\title{
Experimental Research of the Antenna Array with Electronic and Combine Electronic and Mechanical Beam Steering
}

\author{
Ye.A. Litinskaya, A.D. Nemshon, A.V. Stankovsky, S.V. Polenga, Y.P. Salomatov \\ Institute of Engineering Physics and Radioelectronics \\ Siberian Federal University \\ Krasnoyarsk, Russia \\ e-mail: YLitinskaya@gmail.com
}

\begin{abstract}
Experimental research results for antenna array with different types of the beam steering are considered. Amplitude and phase distributions over the antenna array aperture are presented at different beam directions for electronic and combined electronic and mechanical beam steering types. Gain difference between two presented beam steering types was more than $6 \mathrm{~dB}$ at 60 degrees scanning angle.
\end{abstract}

Keywords-electronic beam steering; combined electronic and mechanical beam steering; antenna array.

\section{INTRODUCTION}

Satellite telecommunications in motion are currently a promising task. Antenna arrays with electronic beam steering (EBS) are widely used in mobile communications networks, but such antenna systems have some disadvantages. Main disadvantage of the antenna arrays with electronic beam steering is the limited scanning angle range [1]. The scanning angle range can be increased and antenna system cost can be reduced by using combine electronic and mechanical beam steering. Block diagram of the antenna array with combined electronic and mechanical beam steering (EMBS) is presented in Fig. 1 [2].

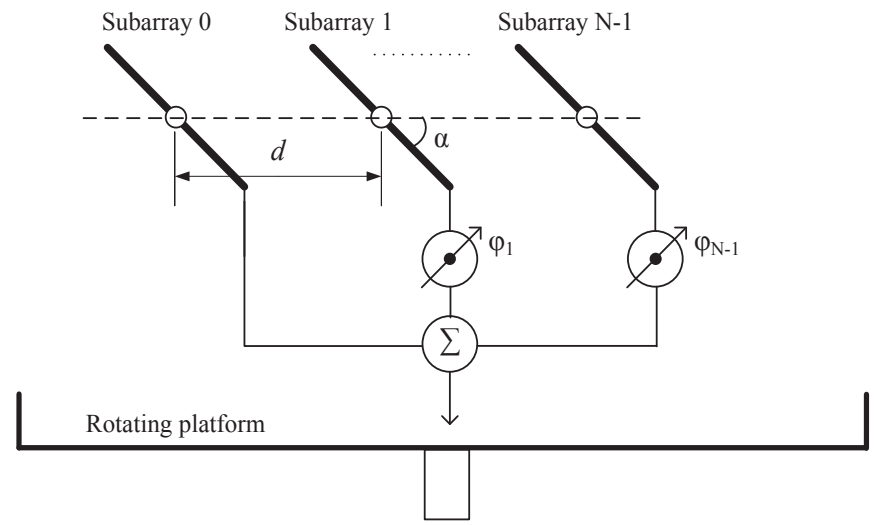

Fig. 1. Block diagram of the phased antenna array with electronic and mechanical beam steering.

Considered antenna array consists of eight subarrays, which can be mechanically rotated around their axis. Subarrays are placed on common platform. Mechanical rotating of the common platform provides scanning in azimuth plane. Scanning in elevation plane is achieved by linear phase delays introduced in the subarrays feed circuits and simultaneous subarrays rotation at angle $\alpha$. The difference between fully electronic beam steering and combined electronic and mechanical beam steering is subarrays mechanical rotation. Each subarray consists of 20x1 patches. The subarray steps are $0,85 \lambda$ in elevation plane and $0,5 \lambda$ in azimuth plane. Antenna array dimensions are $275 \times 225 \mathrm{~mm}$. Experimental research results of antenna array with combined electronic and mechanical beam steering are presented in article [3]. The comparison of calculation and measurement results is presented in article [4]. In this paper we will compare these results.

\section{EXPERIMENTAL RESULTS}

The comparative research results of the antenna array with two different beam steering types are presented in this part.

Antenna array power dividers for different scan angles are presented in Fig. 2. Phase delays are realized by means of different lines length providing necessary delay times. Each subarray is connected to corresponding power divider output.

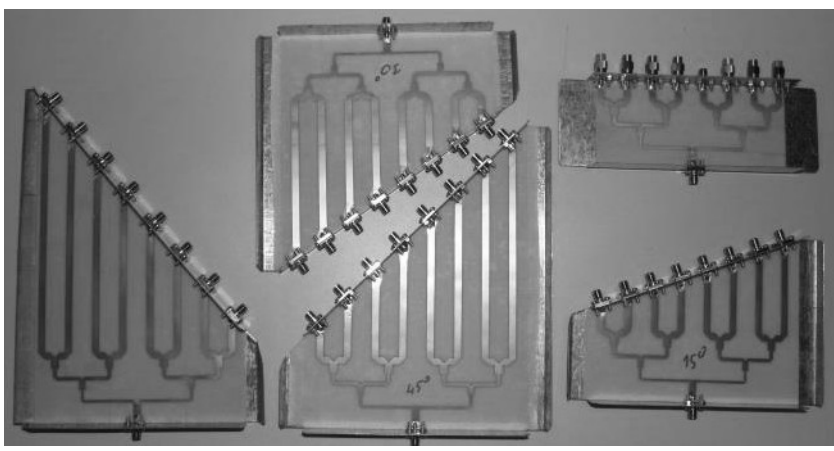

Fig. 2. Power dividers with linear phase delay

The measured antenna arrays with different beam steering types are presented in Fig. 3, 4. 


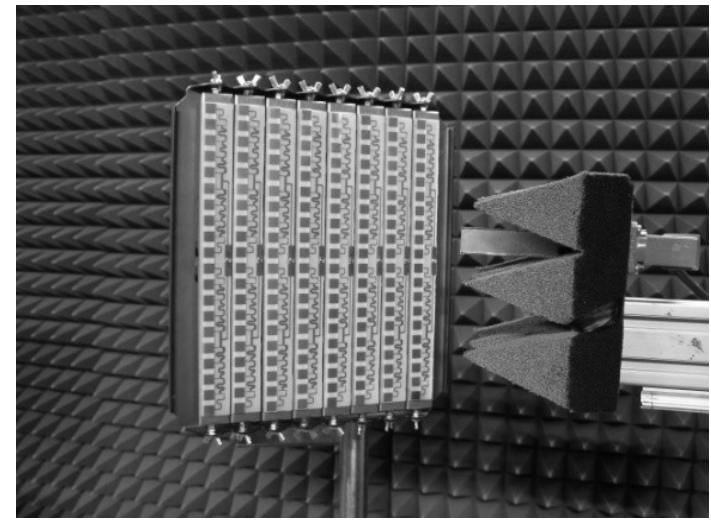

Fig. 3. Antenna array with fully electronic beam steering for 60 degrees scan angle.

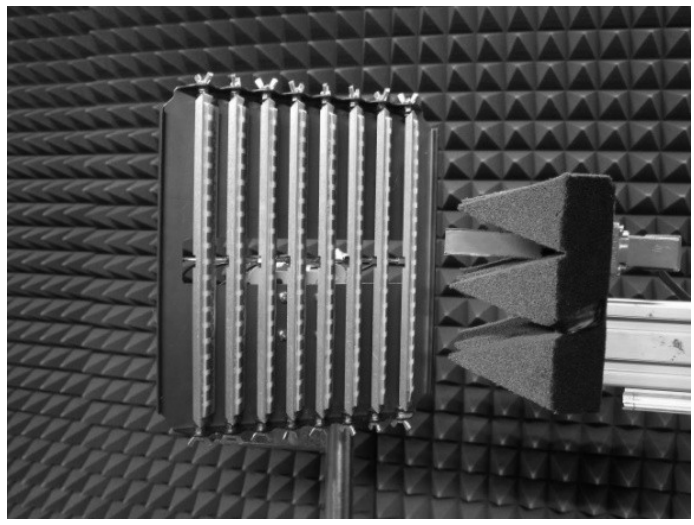

Fig. 4. Antenna array with combine electronic and mechanical beam steering for 60 degrees scan angle.

The same power dividers for EBS and EMBS were used. Subarray rotation was the only difference between these beam steering types.

Measurements were carried out in anechoic chamber by near field planar scanning method. Scanning plane was perpendicular to beam direction to minimize scanning area. Radiation patterns for beam steering angles $30^{\circ}, 45^{\circ}$ and $60^{\circ}$ with using of two beam steering types (fully electronic and combined electronic and mechanical) are shown in Fig. 5-7.

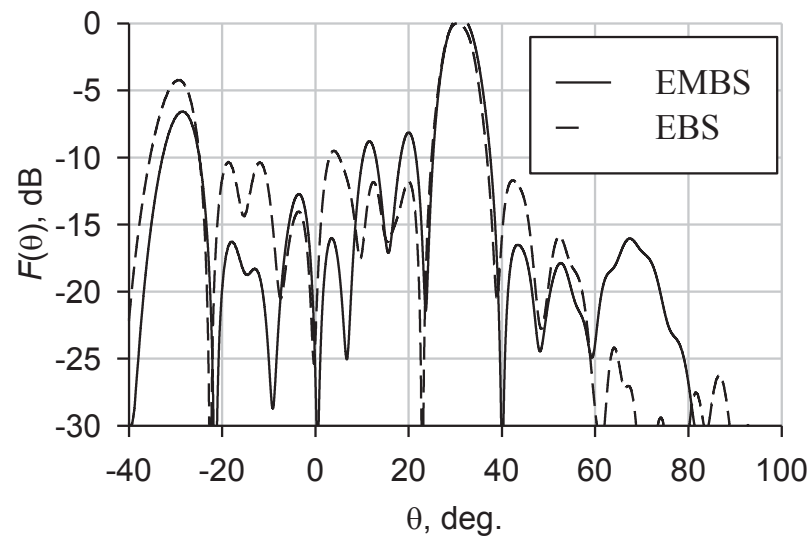

Fig. 5. Radiation patterns for EMBS (solid line) and EBS (dotted line) at $30^{\circ}$ inclination angle.

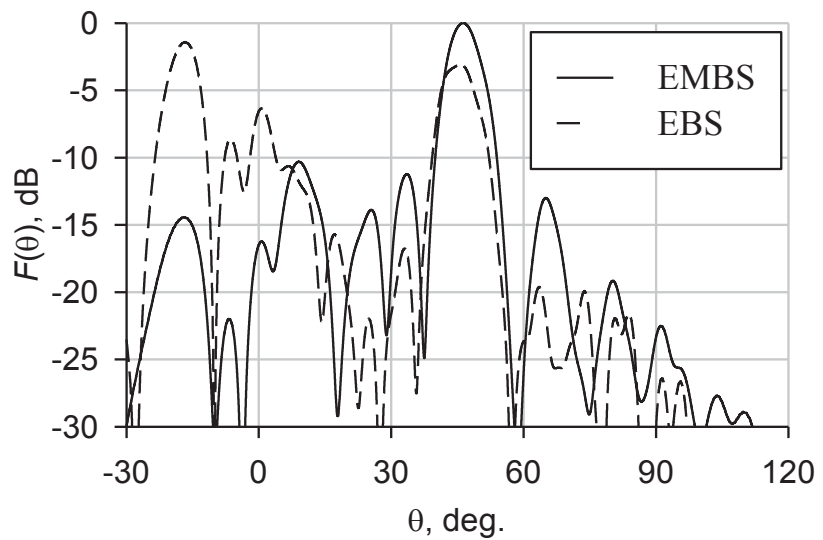

Fig. 6. Radiation patterns for EMBS (solid line) and EBS (dotted line) at $45^{\circ}$ inclination angle.

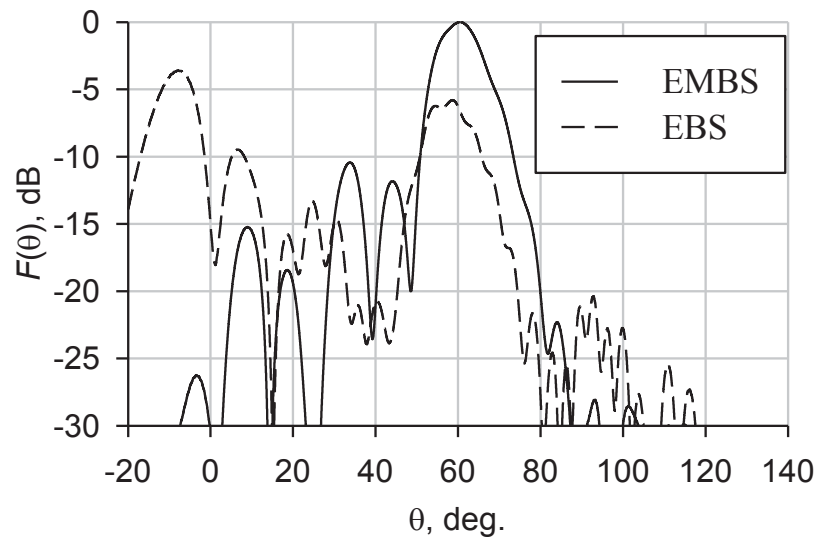

Fig. 7. Radiation patterns for EMBS (solid line) and EBS (dotted line) at $60^{\circ}$ inclination angle.

The EBS antenna directional characteristics significantly degrade with the beam inclination at more than 30 degrees. The EBS antenna gain is $6 \mathrm{~dB}$ less than the EMBS antenna gain for 60 degrees beam steering angle. Effective step of antenna subarrays reduces with beam direction angle increasing, that fact leads to diffraction lobes reduction.

For the EMBS antenna gain reduction as low as $2.5 \mathrm{~dB}$ in overall 120 degrees beam steering range is observed [2].

Amplitude and phase distributions over the EBS and EMBS apertures at 60 degrees scanning angle are shown in Fig. 8-11.

The amplitude and phase distributions of EMBS are flat at 60 degrees beam steering angle in a plane perpendicular to the pattern direction. Distribution distortions are small.

Amplitude and phase distributions of the EBS have significant distortions at 60 degrees beam steering angle. Significant amount of the total power is radiated via side lobes. 


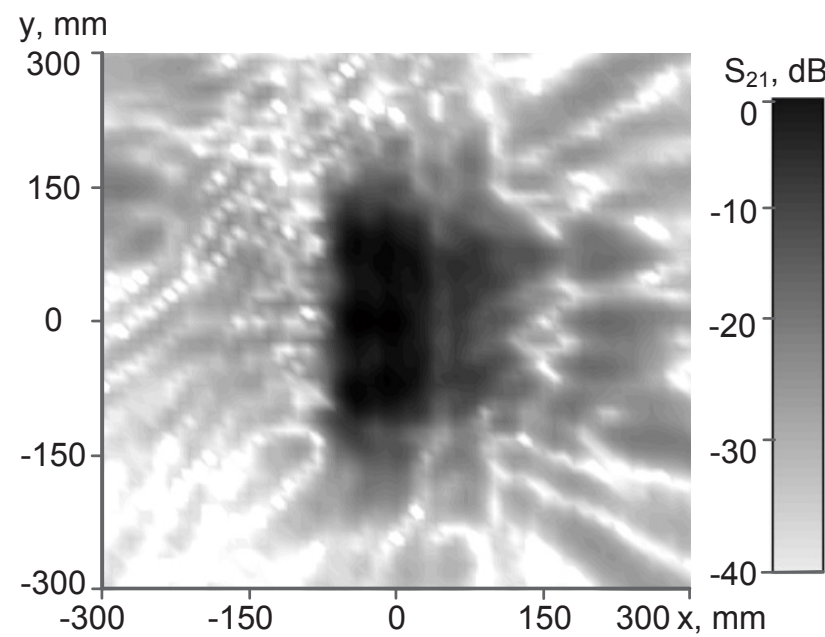

Fig. 8. Amplitude distribution of EMBS at $11 \mathrm{GHz}, 60^{\circ}$ beam direction.

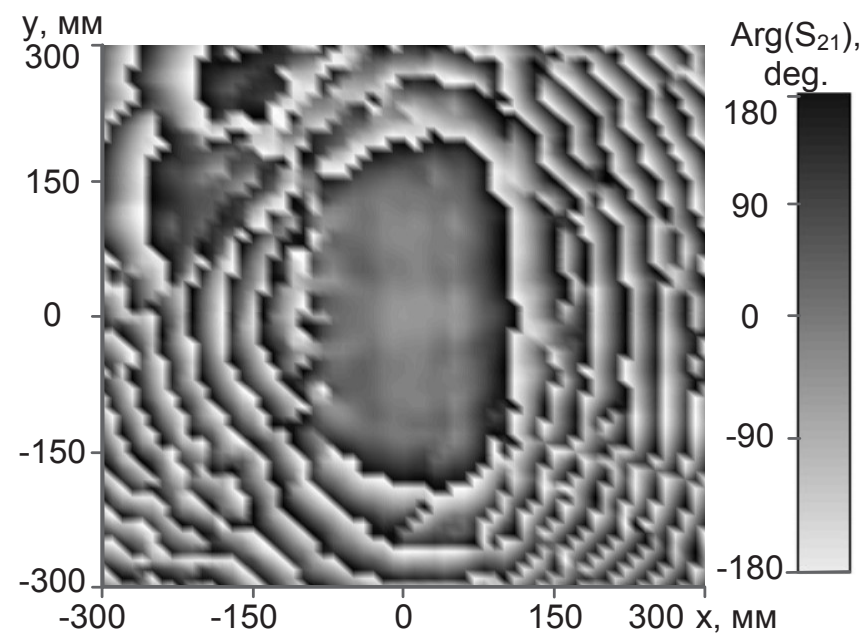

Fig. 9. Phase distribution of EMBS at $11 \mathrm{GHz}, 60^{\circ}$ beam direction.

Research results show advantages of the combined electronic and mechanical scanning method over the electronic one. Gain difference of two presented beam steering types was more than $6 \mathrm{~dB}$ at 60 degrees beam steering angle.

\section{CONCLUSION}

In this paper antenna array design with combined electronic and mechanical beam steering in elevation is presented. Directional characteristics of researched antenna array at different scan angles with using of two beam steering types (electronic and combined electronic and mechanical) are considered. Such antenna type has low cost and weight due to using photolithographic method in manufacturing process. EMBS can be used for mobile satellite communication systems in northern areas of the world, where it is necessary to utilize antenna systems with scanning angles more than 60 degrees.

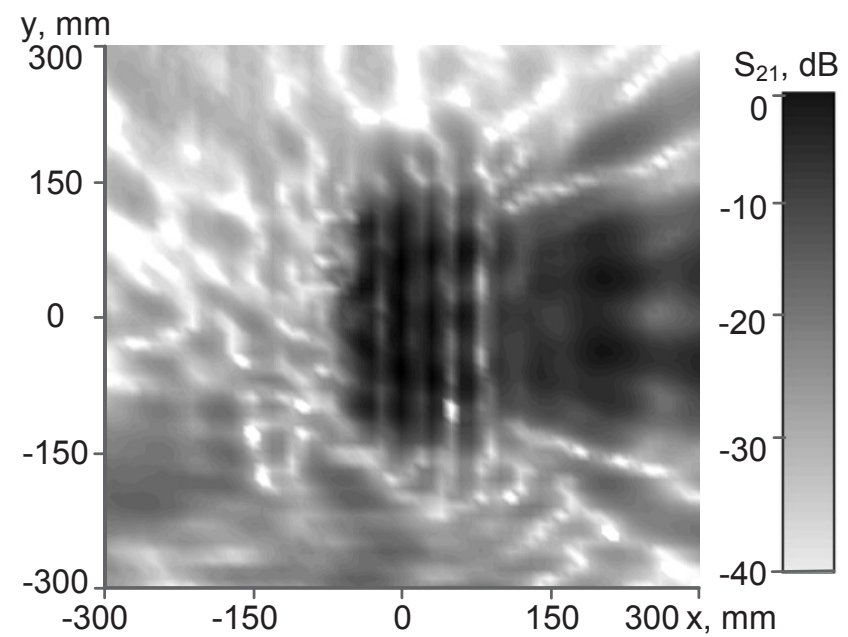

Fig. 10. Amplitude distribution of EBS at $11 \mathrm{GHz}, 60^{\circ}$ beam direction.

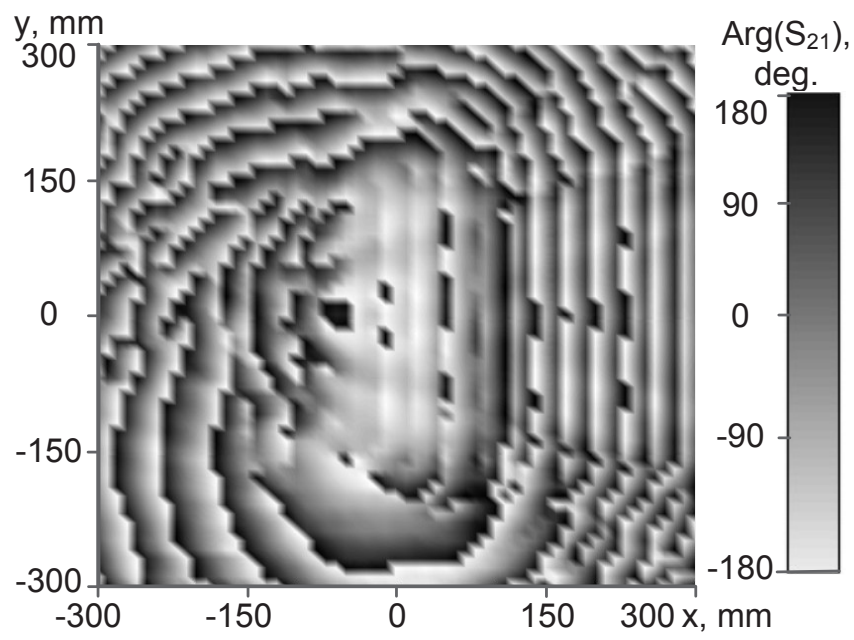

Fig. 11. Phase distribution of EBS at $11 \mathrm{GHz}, 60^{\circ}$ beam direction.

\section{REFERENCES}

[1] Robert C. Hansen, Phased Array Antennas, 2009, John Wiley \& Sons, Inc.

[2] A. M. Alexandrin, K. V. Lemberg, Ye. A. Litinskaya, S. V. Polenga, Y. P. Salomatov // 2013 International Siberian Conference on Control and Communications (SIBCON). Proceedings. - 2013. - P. $52-53$.

[3] Ye. A. Litinskaya, S. V. Polenga, V. S. Panko, Y. P. Salomatov. Phased array antenna with combined electronic and mechanical beam steering// «Uspekhi sovremennoi radioelektroniki» (Achievements of Modern Radioelectronics). - 2015. - No. 1 - P. $24-27$.

[4] Ye. A. Litinskaya, A. D. Nemshon, S. V. Polenga, Y. P. Salomatov The low-profile antenna array with wide-angle scanning //25nd Int. Crimean Conference "Microwave \& Telecommunication Technology" (CriMiCo'2015), -2015. - P. 461-462. 